\title{
Therapie in der Neurologie
}

\author{
Therapeutic Chances in Neurology
}

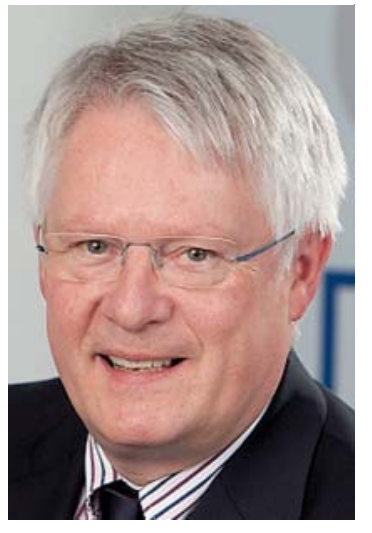

Prof. Dr. med. Heinz Reichmann
Liebe Leserinnen und liebe Leser,

wenn man mit jungen Medizinstudenten spricht und z.B. Studenten im praktischen Jahr auf einer neurologischen Station nach ihren Zukunftsplänen fragt, hört man häufig, dass sie sehr gerne Innere Medizin zu ihrem Spezialgebiet machen wollen. Auf die Frage, warum sie dann in der Neurologie das freiwillige Tertial absolvieren, kommt häufig die Antwort: „Weil man dadurch für die Innere Medizin außerordentlich wertvolle $\mathrm{Zu}$ satzeinblicke gewinnen und Informationen über die faszinierende Welt der Neurologie erhalten kann." Selbstverständlich stellt sich dann sofort die Frage, warum die jungen Kolleginnen und Kollegen nicht Neurologen werden wollen, und die Antworten sind vielfältig. Zum einen gibt es aufgrund der $70 \%$ weiblichen Studenten, ähnlich wie in chirurgischen Fächern, das Problem, dass gerade unsere Mitarbeiterinnen sich vor Schichtdiensten und insbesondere intensivneurologischen Stationen nicht gerade fürchten, aber eine Arbeit unter diesen Bedingungen als nicht besonders familienfreundlich ansehen. Zugegebenermaßen sind z. B. an der von mir geleiteten Klinik im Schichtdienst neben der neurologischen Intensivstation die interdisziplinäre Aufnahmestation und die Schlaganfallstation zu versorgen. Als zweites Argument, warum viele junge Kolleginnen und Kollegen die Innere Medizin vorziehen, höre ich zu meinem Erstaunen immer wieder, dass man dort wesentlich aktiver und effektiver therapieren könne. Hier setzt nun das vorliegende Editorial an, weil ich diese Sichtweise der jungen Kolleginnen und Kollegen nicht teilen kann. Die Neurologie ist mittlerweile von einem deskriptiven Fach zu einem Fach geworden, das mittels modernster Verfahren (waren es früher insbesondere neurophysiologische Methoden, haben wir heute darüber hinaus die morphologische und die funktionelle Bildgebung sowie die Neurogenetik) neue Türen zur noch sorgfältigeren Diagnostik der neurologischen Erkrankungen geöffnet hat. Entscheidend ist aber, dass aus diesen Erkenntnissen in vielen Gebieten der Neurologie neue und faszinierende Therapieoptionen erwachsen sind. Ich möchte dazu nur drei Beispiele nennen, die auch die jungen Kolleginnen und Kollegen überzeugen müssten, dass eben auch die Neurologie beeindruckende therapeutische Erfolge ermöglicht. Während der vergangenen Monate sind vier Studien im Rahmen der Schlaganfallforschung vorgestellt worden, die eine intraarterielle interventionelle Lyse-Therapie mittels Stent-Retrievern der alleinigen intravenösen Lyse-Therapie als deutlich überlegen gezeigt haben. Ich gehe davon aus, dass etwa $10 \%$ aller Schlaganfallpatienten von dieser interventionellen Therapieoption profitieren und ein noch bei Weitem besseres neurologisches Ergebnis erzielen werden, als dies bislang schon unter der intravenösen Lyse-Therapie möglich war. Nachdem die Schlaganfallbehandlung in den meisten neurologischen Kliniken zum Hauptaufgabenfeld geworden ist, ist jeder Fortschritt in dieser Disziplin von besonderer Bedeutung.

Ein zweites Beispiel ist die tiefe Hirnstimulation. Früher waren z.B. Parkinson-Patienten in fortgeschrittenen Stadien unmenschlichen On-offSituationen ausgeliefert und auch Neurologen konnten an diesem Jo-Jo-Effekt sehr wenig ändern. Mittels Pumpentherapie wie DuodopaPumpe und Apomorphin-Pumpe besteht jetzt die Möglichkeit, durch eine hohe kontinuierliche Dopaminrezeptorstimulation den Patienten in den fortgeschrittenen Stadien der Parkinson-Erkrankung nachdrücklich zu helfen. Noch effektiver und auch früher verwendbar ist die tiefe Hirnstimulation, die weltweit in den meisten Zentren im Nucleus subthalamicus vorgenommen wird und über deren Indikation und Wirksamkeit kaum mehr Zweifel bestehen. Mittlerweile hat sich die tiefe Hirnstimulation aber auch Patienten geöffnet, die unter unerträglicher und bisher kaum therapierbarer generalisierter Dystonie leiden oder auch schwere fokale Dystonien aufweisen. Weitere Krankheitsbilder sind die Tic-Erkrankungen, und wenn man in unsere Schwesterdisziplin, die Psychiatrie, schaut, scheint die tiefe Hirnstimulation auch eine Option bei schweren Depressionen und Zwangserkrankungen zu werden.

Ein drittes Beispiel sind die bisher nicht behandelbaren destruktiven Myopathien, das heißt, genetische Formen der Muskeldystrophien wie Duchenne, Becker, myotone Muskeldystrophie, Gliedergürteldystrophie und myotone Dystrophie. Mithilfe des sogenannten Exon Skipping ist man mittlerweile in der Lage, bei Duchenne-Knaben einzelne Teile der pathologischen Gensequenz abzuschalten und so ein teilfunktionierendes Dystrophin-Protein in den Kindern zu produzieren. Erste Erfolge im Bereich der Gliedergürtelmuskeldystrophie betreffen das sogenannte Dysferlin, dessen genetische Basis bei den Patienten zu einem Abbruch des Leserasters führt. Es ist jetzt gelungen, dem gegenzusteuern und zumindest teilweise funktionierendes Dysferlin in erkrankten Patienten herzustellen.

Eine letzte Option, die ich hier nennen will, ist das sogenannte Myostatin. Für uns alle ist das Wachstum des Körpers und der Muskulatur vorprogrammiert irgendwann beendet. Eine wesentliche Rolle spielt dabei das Myostatin. Es ist jetzt 
mehreren Forschern und Firmen gelungen, bei Patienten und Labortieren mit Muskeldystrophien oder Critical-Illness-Myopathie das Myostatin auszuschalten und so zu einer Zunahme der Muskulatur und dadurch zu einer Zunahme der Muskelkraft zu kommen.

Diese wenigen Beispiele zeigen, welchen Siegeslauf die Therapie in der Neurologie nimmt, und wir können davon ausgehen, dass sich auch bei den zwei schweren Erkrankungen, dem Glioblastoma multiforme (bei dem sich durch Voruntersuchung gewisser Gene neue Therapieoptionen eröffnet haben) und der amyotro- phen Lateralsklerose (bei der z.B. in einem Paper vor Kurzem beim kombinierten Einsatz von Valproinsäure und Lithium positive Effekte gesehen wurden), ebenfalls in absehbarer Zeit deutliche Therapiefortschritte zeigen werden. Wir sollten somit die besten jungen Medizinstudenten ermutigen, unser faszinierendes Fach zu ergreifen, ohne zu vergessen, darauf hinzuweisen, dass den Studenten, die am Gehirn Interesse haben, die Fächer Psychiatrie, Neuropädiatrie, Neuroradiologie, Neuropathologie und viele mehr als Optionen offenstehen. 